\title{
La mélancolie. Le Vent
}

Jean-Pierre Daumard

\section{(2) OpenEdition}

Journals

Édition électronique

URL : http://journals.openedition.org/ccs/635

DOI : $10.4000 /$ ccs. 635

ISSN : 2558-782X

\section{Éditeur :}

Presses universitaires de Rennes, Association des lecteurs de Claude Simon

\section{Édition imprimée}

Date de publication : 31 décembre 2009

Pagination : 47-66

ISBN : 9782354120580

ISSN : 1774-9425

\section{Référence électronique}

Jean-Pierre Daumard, "La mélancolie. Le Vent », Cahiers Claude Simon [En ligne], 5 | 2009, mis en ligne le 21 septembre 2017, consulté le 19 avril 2019. URL : http://journals.openedition.org/ccs/635 ; DOI : $10.4000 /$ ccs. 635 


\title{
Lectures $d u$ Vent
}

\section{La mélancolie Le vent}

\author{
Jean-Pierre DAUMARD
}

\section{Mélancolie et baroque}

Claude Simon n'est guère enclin à connaître la mélancolie. Point de disposition chez lui à l'humeur noire ni de goût pour la langueur, la mièvrerie ou la fadeur des représentations traditionnelles de la mélancolie,

[...] ces images plus ou moins mièvres à la mode chez les préraphaélites anglais, aux couleurs fades, de femmes languissantes, à l'œil rêveur, ou encore, en mettant les choses au mieux, de cet ange pensivement assis, drapé dans une longue robe, soutenant sa tête d'un poing [...] $(J P, 1122)$

Point d'inclination pour la psychopathologie de la mélancolie, point de goût pour l'esthétique traditionnelle de la mélancolie.

C'est pourtant ce terme de mélancolie, si « ambigu, à malentendus » qu'il considère le mieux apte à exprimer l'expérience limite de 
la plus avide protestation de la vie face à l'imminence de la mort ${ }^{1}$. La mélancolie ainsi entendue serait bien l'expérience oxymorique par excellence de la vie qui ne s'affirme comme telle que de se détacher du fond de la mort. Expérience essentielle et sans pathos, métaphysique, d'une sorte de " proximité de l'éternel » et d'avidité au "recueillement de l'essence », comme dit Romano Guardini, mais dans une perspective et dans des termes spiritualistes qu'évidemment Claude Simon ne partage pas $^{2}$. La mélancolie ainsi comprise serait le sentiment le plus vif de toucher à l'essence même de la vie, dans sa plus intime et indicible contradiction.

De là découle le baroque, entendu, pour commencer, dans le sens large que lui donne Jacques Derrida, celui de " réponse - calculée mais embarrassée, ludique et élusive -, aux apories du tact $»^{3}$. Autrement dit : celui d'un toucher à l'essence et à l'essentiel en s'en écartant. De l'aporie de la mélancolie découle alors, "ludique et élusive ", la profusion baroque. D'où ce titre : "La mélancolie Le vent", dans la contiguïté et l'engendrement des deux termes : la mélancolie, graine et fruit de l'écriture du roman Le Vent. L'absence de détermination entre « mélancolie » et " vent», pour signifier la mélancolique déliaison d'une appartenance, en lui donnant l'allure du « cas régime absolu » de l'ancien français.

Ce pourrait être une façon de suggérer cette sorte de déhiscence qu'est dans Le Vent de Claude Simon, à la fois l'expérience divisante du moi (du personnage principal, Montès), de sa mélancolie proprement métaphysique, et l'engendrement des mots, des voix, des récits du roman, leur prolifération foisonnante et « baroque " pour tenter, précise le sous-titre, mieux encore que de la raconter, de la restituer.

\footnotetext{
${ }^{1}$ "Il décrit cette déchirante et mélancolique avidité avec laquelle le condamné regarde autour de lui le monde (le reflet du soleil sur le bulbe doré d'une église, les maisons, les gens) qui va continuer d'exister alors que dans quelques instants lui-même ne sera plus rien. » (JP, 1124).

${ }^{2}$ Romano Guardini, De la mélancolie, Seuil 1952, p. 65.

Jacques Derrida, Le toucher Jean-Luc Nancy, Galilée 2000, p. 152.
} 


\section{La mélancolie de la tentative ou de l'essai}

"Tentative de restitution d'un retable baroque ": tel est le soustitre du roman. Le terme de "tentative » ainsi affiché situe d'emblée le roman du côté de l'essai, et suggère aussitôt qu'il aura le caractère pensif, méditatif, voire la démarche tâtonnante qu'on peut en attendre depuis les Essais de Montaigne. Il justifie aussi qu'après coup, plus qu'à la matière romanesque proprement dite, le lecteur méditatiflui-même porte son attention et son intérêt sur ce qui devient l'essentiel : l'approche progressive, insistante, obstinée, d'une pensée de la mélancolie (comme, inversement, d'une mélancolie de la pensée).

Le Vent de Claude Simon apparaît bien comme une "tentative" baroque et mélancolique au sens, cette fois, où Benjamin peut associer les deux termes : baroque parce que faite de « débris dispersés, incomplets » $(V, A)$, mélancolique parce que la restitution de l'unité originaire de " ce qui s'est passé » est à l'évidence parfaitement illusoire, aussi illusoire que la restauration d'un " miroir » brisé : « $[\ldots]$ tenter de rapporter, de reconstituer ce qui s'est passé, c'est un peu comme si on essayait de recoller les débris dispersés, incomplets, d'un miroir [...] ». On voit qu'ici le saut du « débris » ou de la ruine à l'unité perdue est, comme dans l'allégorie, infini, abyssal. Benjamin a bien souligné la différence essentielle entre symbole et allégorie. Autant le symbole " recueille le sens [...] à l'intérieur de lui-même ", autant l'allégorie «se plonge dans l'abîme qui sépare l'image de la signification $»^{4}$. Autant le symbole est $"$ instant mystique $»^{5}$, autant l'allégorie est dialectique suspendue, à l'arrêt, sans solution, sans relève. D'où sa parenté avec le baroque, celui-ci et celle-là pris dans la dispersion de significations sans fin.

Par ailleurs, le «miroir» lui-même, instrument du reflet, de la représentation, peut apparaître comme la métaphore d'une mimesis impossible, mais impossible surtout parce que mal comprise : en termes de restauration, de " restitution " parfaites, intégrales, sans reste, d'un état antérieur. Alors que la mimesis, loin d'être synonyme

\footnotetext{
${ }^{4}$ Walter Benjamin, Origine du drame baroque allemand, Flammarion, "Champs », 1985, p. 178 .

${ }^{5}$ Ibid., p. 178.
} 
de pure et simple imitation ${ }^{6}$, reproduction à l'identique comme serait une reprographie, inscrit en son cœur l'écart et la différence. Or c'est bien là encore qu'est la mélancolie, dans cette tension constante entre l'exigence d'une exacte représentation et le constat à peu près tout aussi constant de son échec ${ }^{7}$.

La raison en est dans cet autre abîme qui existe entre la vie, son " foisonnement désordonné », et ce qui, ordinairement, prétend la reproduire en mots, la relater :

[...] la vie reprend sa superbe et altière indépendance, redevient ce foisonnement désordonné, sans commencement ni fin, ni ordre, les mots éclatant d'être de nouveau séparés, libérés de la syntaxe, de cette fade ordonnance, ce ciment bouche-trou indifféremment apte à tous usages $[\ldots] .(V, 137)$

Hiatus douloureux des mots aux choses, qui ne se console pas d'être grossièrement colmaté. Si donc la « restitution» n'est plus à escompter, reste la « tentative ». Mais cette " tentative " elle-même voit inscrit dans son propre mot le signe de son échec. De fait, entreprise de toucher ce qui ne saurait l'être, dans l'éloignement qu'est tout langage, elle ne peut au mieux qu'errer, à l'image même de la vie : " [...] cette errance, nous-mêmes ballottés de droite et de gauche, comme un bouchon à la dérive, sans direction, sans vue, essayant seulement de surnager et souffrant, et mourant pour finir, et c'est tout... » $(V, 4)$.

Alors, autour du motif éminemment baroque en lui-même du vent (dans son immatérialité tournoyante qui ne peut être appréhendée que par ses effets, tout comme le sublime en peinture), un sujet sans qualité, divisé sans remède ${ }^{8}$, inapte à la vie sociale et au «bonheur », est saisi dans la " restitution» impossible de son histoire, dans une " tentative " par essence inaboutie, et donc " baroque " déjà,

\footnotetext{
${ }^{6}$ " [ ] la mimesis, [...] cette notion à laquelle on garde son nom grec afin de lui éviter la confusion avec l'imitation, la simple et comme on le dit si souvent "servile" imitation." (J.-L. Nancy, Le plaisir au dessin, Editions Hazan, 2007, p. 30).

${ }^{7}$ Il serait assez vain de faire l'inventaire de toutes ces expressions qui disent l'effort assez souvent déçu mais nécessaire, de se représenter: "Et sans doute faut-il essayer de se le représenter, seul $[\ldots] »(V, 55)$.

${ }^{8} \mathrm{Ce}$ que souligne le clivage personnage (Montès)/narrateur, l'un alter ego de l'autre, dans une division et une indistinction parfois troublantes.
} 
au sens trivial du terme d'irrégularité et d'imperfection ${ }^{9}$. Comme si l'écriture, essentiellement déceptive, ne pouvait qu'être le produit d'une déception majeure, d'une mélancolie majeure ${ }^{10}$ et sans recours produisant cet ethos particulier qu'on pourrait dire de détachement, qui affecte le regard porté sur le monde et sur l'Histoire.

\section{La mélancolie du regard porté sur le monde et sur l'Histoire}

\section{Le regard détaché d'un être déconnecté}

Le regard que porte le personnage principal, Montès, sur luimême est le regard d'un personnage déconnecté . " [...] toujours, me dit-il, s'agissant de lui, comme s'il assistait à ses propres actions de l'extérieur, comme déconnecté » $(V, 156)$. De là, peut-être, cette tension identificatoire entre le narrateur et le personnage, dès les premières pages du livre : "Il me semblait donc le voir, assis là, dans ce même fauteuil où je me trouvais moi-même $[\ldots]$ » $(V, 5)$. Reste que de ce double détachement et de cette aptitude à percevoir toutes sortes de dissociations ${ }^{11}$, va naître l'essentiel : un regard toujours détaché. Détaché en ce qu'il n'est jamais que lacunaire et partiel, fragmenté, mais aussi en position de retrait, voire d'« époché » phénoménologique. Il est véritablement détaché dans tous les sens du terme, c'est-à-dire affecté d'un détachement aussi bien physique qu'affectif et intellectuel. Désaffecté en quelque sorte, suspendu, rompant le fil du temps et du sens. Regard qui n'exprime rien, qui ne signifie rien, comme en décrivait Faulkner quand il évoquait dans L'Intrus une " expression sans aucun sens ni passé $»^{12}$.

Il s'agit donc d'un regard littéralement $a b s o l u^{13}$ sur les choses, lesquelles semblent n'être véritablement choses que déliées du temps, de l'histoire, du sens, se détachant sur fond - sinon de néant, le mot trop substantialiste est aussi trop pathétiquement connoté - du

\footnotetext{
${ }^{9}$ Ainsi parle-t-on couramment de « perles baroques ».

${ }^{10}$ Majeure aussi au sens musical du terme. Bien souvent la tonalité du roman a l'éclat, la force jubilatoire, emphatique et polémique parfois, d'une tonalité majeure, pas la tristesse alanguie d'une tonalité mineure.

"« [...] comme si voix et personne faisaient deux, chacune se mettant à vivre d'une vie indépendante $[\ldots] »(V, 118)$.

${ }^{12}$ William Faukkner, L'Intrus, Gallimard, "Folio », p. 229.

${ }^{13}$ Dans le sens le plus étymologique du terme : absolvere, détacher.
} 
moins de vide. Montès, le personnage central du roman de Claude Simon, a le regard détaché de celui qui, inapte à entrer dans la logique des liens (à commencer par celle des liens sociaux), passe alors aux yeux et dans le discours des autres, pour un « idiot »: " Un idiot, voilà tout. Et rien d'autre " $(V, 3)$. Voilà comment est proférée dès l'incipit du roman et par le biais de la voix normative, socialisée et sans appel du notaire, l'incompréhensible acédie ${ }^{14}$ de Montès.

Le regard de Montés, détaché, désarrimé de tout sens, sera également celui qu'Hélène pose sur une scène muette de dispute dans le bureau de son père :

[...] car à travers la porte vitrée aucun bruit ne lui parvient, et de ce fait la scène ayant ce on-ne-sait-quoi d'insolite, d'angoissant et d'absurde, comme lorsqu'une panne de son prive tout à coup de la parole les personnages d'un film et qu'on les voit néanmoins continuer à s'agiter et à vivre $[\ldots] .(V, 129)$

Voilà pourquoi un tel regard peut se faire si souvent pictural, convertissant le spectacle du monde en une série de tableaux minutieusement cadrés et découpés, parfaitement détachés de tout, mélancoliques comme des tableaux de Hopper. Ainsi :

[...] de l'autre côté de la place cette dernière boutique encore allumée, insolite dans la nuit, trop loin pour qu'il pût entendre, saisir autre chose que cette fraction muette de vie s'inscrivant dans le rectangle lumineux que découpaient les vitres de la devanture [...]. Cela. Comme, dit-il, une boîte, une sorte de petit théâtre lumineux au sein de la nuit, avec ses personnages muets, dessinés et coloriés avec cette absurde et minutieuse précision des détails qui contribuait à les rendre irréels, privés d'atmosphère, ciselés. $(V, 75-76)$

La mélancolie ici n'est rien d'autre que retrait de l'action au profit de la pure contemplation à la fois solitaire, avide et fascinée, semblable à celle de l'« homme des foules» de Baudelaire ${ }^{15}$.

\footnotetext{
${ }^{14}$ Une " acédie " particulière, dont le fond n'est plus l'être, mais ce "vent sauvage et froid " $(V, 8)$, dans une sorte de réadaptation et de radicalisation contemporaines de l'« acidiosus » médiéval.

${ }^{15}$ Cet Homme des foules est d'abord un tableau : « Vous souvenez-vous d'un tableau (en vérité c'est un tableau !) écrit par la plus puissante plume de cette époque ? ", et le tableau d'un homme en proie à une curiosité fascinée : «Finalement, il se précipite à travers cette foule à la recherche d'un inconnu dont la physionomie entrevue l'a en un clin d'oeil, fasciné. La curiosité est devenue une passion fatale, irrésistible " ( Le peintre de la vie moderne », Euvres complètes, II, Gallimard, «Bibliothèque de la Pléiade, 1976, p. 689-690).
} 
D'où l'oisiveté de Montès qui assiste à sa vie plutôt qu'il ne la vit : « [...] assistant impuissant, navré et ironique au déroulement de sa propre vie ou plutôt de celle qu'un autre lui-même lui imposait [...] » (V, 56). On pense ici au Dostoïevski des Carnets du sous-sol qui voyait dans l'homme d'action " une créature essentiellement limitée » mais dans l'homme du regard, de la contemplation, donc de la pensée, un être pris dans l'enchaînement des "causes premières " qu'il fait indéfiniment reculer : «Je m'exerce à penser ; par conséquent chez moi toute cause première en fait immédiatement surgir une autre, plus première encore, et ainsi de suite, à l'infini. $»^{16}$. De même Montès et le narrateur du roman, l'un et l'autre pris dans ces successions de regards lacunaires et détachés certes, mais entraînés parfois dans un regard surplombant, démesuré, ouvert sur un infini aussi bien spatial que temporel, littéralement hyperbolique, proche de ce que Barthes appelle le numen : " excès de pose [...], hystérie figée, éternisée, piégée, puisqu'enfin on la tient immobile, enchaînée sous un long regard. $»^{17}$. Un « long regard » qui n'est pas sans évoquer l'« œil de Dieu» de toute la tradition baroque, dans le transport mélancolique qu'il expose, un " long regard » qui ne diffère guère d'une sorte d'extase.

\section{L'extase mélancolique et baroque}

Un passage du chapitre V relate le " quelque chose de bizarre " $(V, 75)$ ressenti par Montès : le sentiment que « [sa] conscience passait alternativement au dehors et au dedans de [lui] », qui le fait se percevoir simultanément regardé et regardant puis, dans une sorte d'hyperbolique travelling arrière, aussi bien spatial que temporel, appréhender la ville, ses habitants occupés à se reproduire, " la campagne, les chemins, les trains, et encore les autres villes", le tout culminant dans une sorte d'hallucination ou de semblant d'apothéose baroque où semblent fusionner la chair de Rose et celle du monde

\footnotetext{
${ }^{16}$ Dostoïevski, Carnets du sous-sol, Babel, 1993, p. 28.

${ }^{17}$ Roland Barthes, "Le numen ", Roland Barthes par Roland Barthes, Seuil, "Écrivains de toujours », 1980, p. 138.
} 
« [...] et de tout cela il me semblait aussi qu'on pouvait entendre comme une respiration, comme celle de sa chair à elle, cette même palpitation secrète, multiple, mystérieuse, parce que la chair du monde est femelle par ce fait qu'elle est capable d'engendrer et de créer pour ainsi dire sans même s'en apercevoir... ». $(V, 75)$

Extase baroque ici, non seulement ce renversement de perspective (regardant/regardé), mais cet empiètement de la vision et du mouvement dans une " folie du voir " qui fait du "visuel affranchi du seul cadre optique représentatif $»^{18}$, une sorte de nouvel avatar de l' " œil de Dieu ». Baroque et mélancolique, ce transport hyperbolique, cette tension allégorique qui, passant de la " chair» de Rose à " la chair du monde ", opère un " glissement du visuel au visionnaire ", comme Patrick Longuet l'a justement remarqué, et confine à ce qu'il appelle "l'emphase statufiante de l'allégorisme $\|^{19}$. Dans ce passage, la figure allégorique enfle et prolifère en accord avec la propre prolifération de la « chair du monde [...] capable d'engendrer et de créer pour ainsi dire sans même s'en apercevoir ». Sans doute le propre de l'allégorie est-il de toujours s'excéder, mais ici son excès joint à son détachement semble culminer dans la stase même de sa démesure, en transport mi-épique mi-épiphanique qui, pour finir, s'inachève dans le suspens du point de suspension. Toujours est-il qu'ici l'on perçoit nettement cette "parenté entre la tristesse et l'ostentation », si caractéristique des « formations verbales du baroque » pour Benjamin ${ }^{20}$.

À l'opposé du transport hyperbolique cette fois, autre avatar modeste et désacralisé de l'« œil de Dieu » baroque : l'appareil photographique, outil favori du narrateur, de Montès et de Claude Simon. L'« appareil photo d'au moins cent mille francs », seul objet de valeur que Montès porte sur le ventre, qui tranche sur son accoutrement de clochard, est aussi l'emblème du mode de représentation élu par Claude Simon car il permet l'enregistrement ponctuel, exact, du réel et du contingent pur, dégagé de toute temporalité signifiante, dans la mélancolie du suspens. Pour Barthes, rien de plus significatifde la mélancolie moderne, de la " Mort asymbolique, hors religion, hors

\footnotetext{
${ }^{18}$ Catherine Buci Glucksmann, La folie du voir. De l'esthétique baroque, Galilée, 1986, p. 70 .

${ }^{19}$ Patrick Longuet, Lire Claude Simon, La polyphonie du monde, Minuit, 1995, p. 37.

${ }^{20}$ Walter Benjamin, op. cit., p. 151.
} 
rituel, sorte de plongée brusque dans la Mort littérale " ou "plate ", sans catharsis ${ }^{21}$, que la photographie. Le déclic photographique est pure saisie de l'instantané La Vie/la Mort dont reste la mélancolie d'un «ça a été $\|^{22}$.

Nombreux sont les " ça a été " dans Le Vent. Ils semblent encore plus mélancoliques quand ils exposent une saisie (immobile donc) du mouvement. Ainsi cette sorte de cliché (clic/clac) de " cheveux très noirs qu'un coup de vent au moment de la photo tordait et rabattait sur la figure » $(V, 40)$. Mélancolie de l'impossible restitution du mouvement comme mouvement, et de sa retombée en geste. À rattacher peut-être à la nostalgie de « ce qui ne bouge pas », dans de "vieilles gravures de la ville ». Ajoutons que le régime du "ça a été » est très exactement celui de l'économie narrative du roman. Peu de diégèse pure en effet, laquelle n'est jamais exempte de tonalité épique, mais plutôt une suite de " ça a été " et tout particulièrement de "ça a été dit $»$. Somme de témoignages dont le narrateur semble se borner à tenir le registre, un registre augmenté du produit de ce qu'il imagine. Dans la posture de l'ange de Diirer, finalement !... Or, c'est bien le regard dans son excès, aussi bien son excès de détachement au sens de fragmentation que l'excès de désarrimage, qui dévalue l'action, l'intrigue et l'Histoire.

21 Roland Barthes, La Chambre claire, Seuil, Euvres complètes, tome V, 1977-1980, pp. 861 et sq.

${ }^{22}$ Ibid., p. 863. Mireille Calle-Gruber oppose à cette conception de la photo comme saisie de la mort, cette belle idée de " l'affleurement du monde dont les éléments font surface " (Le Grand Temps. Essai sur l'œeuvre de Claude Simon, Septentrion, 2004, p. 61). Il est vrai aussi que Barthes lui-même, plus haut dans son ouvrage (p. 854), imagine qu'en latin, " photographie » se dirait : " imago lucis opera expressa », c'est-à-dire : image révélée, « sortie ", « montrée », « exprimée " (comme le jus de citron) par l'action de la lumière, et il souligne tout ce que cette conception de la photographie a de vivant. Mais tout cela n'enlève rien à la tonalité baroque de l'opération, puisque le propre du baroque est peutêtre bien cette venue du fond qui affleure la surface (ainsi du fameux rideau rouge de $L a$ mort de la Vierge du Caravage) et n'est-ce pas de cette façon de « faire surface » que s'ouvre le battement mélancolique ? "Affleurement de l'être ", "intériorité qui ne peut avoir lieu qu'à la surface ", dit encore Mireille Calle-Gruber, mais tout cela ne correspond-il pas à cette mélancolie sèche, plate et sans pathos qu'est simplement la saisie de l'écart, ce qu'elle appelle aussi « la fêlure constitutive de la comparution»? 


\section{Déception mélancolique des histoires}

Claude Simon procède en effet à une rapide dépréciation du drama de l'action, de l'action dramatique, dès le début du roman. Les événements que le " héros » Montès aura déclenchés dans la ville, ou plutôt " débridés ", étant le fait, bien plus que de ses actes, de sa " seule apparition ", de sa " seule présence, à la façon de ces réacteurs chimiques " $(V, 4-5)$. Le narrateur s'emploie, par le biais du notaire, à ruiner l'intérêt et même l'existence des situations dramatiques, d'un romanesque qui se réduirait au modèle dramatique :

[...] j'ai lu une fois qu'on avait dénombré trente-deux ou trente-six situations théâtrales. Laissez-moi rire. Parce qu'avec les cinq doigts de la main je vous garantis que vous avez amplement de quoi compter les différents cas auxquels tout peut se ramener et même avec un seul, $[\ldots]$ parce que l'unique mobile de toutes les actions humaines, de tous les prétendus drames psychologiques, [...] eh bien c'est l'intérêt $[\ldots] .(V, 6)$

Le romanesque de l'action au sens dramatique est d'autant plus dévalorisé que Claude Simon semble avoir voulu y reprendre, ironiquement, les ingrédients les plus éculés du drame, voire du mélodrame $^{23}$ (vol, jalousie, amour impossible, crime...) pour les réduire à néant. Ironie supplémentaire : c'est chez un notaire, un notaire en possession de l'« état civil » (clin d'œil humoristique et ironique à Balzac $[\mathrm{V}, 8]$ ), que se donne à entendre la vacuité du poncif dramatique. Voilà pourquoi tout récit est

[...] faux, artificiel, comme est condamné à l'être tout récit des événements fait après coup, de par le fait même qu'à être racontés les évènements, les détails, les menus faits prennent un aspect solennel, important que rien ne leur confère sur le moment. $\{V, 35)$

Vanité des histoires et des récits qui sont réorganisés, a posteriori, par les exigences de la raison. Un tel constat mélancolique n'est finalement pas si éloigné de celui de Sartre-Roquentin dans $L a$ Nausée! De plus, la mélancolie du pur regard est augmentée de la

${ }^{23}$ Carl Gustaf Bjurström va même jusqu'à parler de « vieilles règles du feuilleton » (Critique, novembre 1981, p. 1157.) 
conscience, ce "petit quelque chose en plus juste pour son malheur " $(V, 36)$, quelque chose en plus comme le " secret douloureux » baudelairien : mélancolie de la conscience d'être faille ou déficit ontologique. D'où, entraînée avec la dépréciation de l'histoire et du récit traditionnels, la dépréciation de l'Histoire.

\section{Perception mélancolique et baroque de l'Histoire}

D'une part l'Histoire semble bien souvent se diluer dans le " grand temps » de la géographie et d'une immémoriale répétition (ainsi de ces peuples du bassin méditerranéen qui s'étripent depuis la création du monde [ $V, 107])$, d'autre part elle est aussi perçue comme une dégradation, comme un "déclin inéluctable $\|^{24}$. De toute part elle est mélancolique : mélancolie de la stase, de la « rigidité tétanique de la contemplation $»^{25}$, mélancolie de la ruine.

Mélancolie de la dégradation de l'Histoire de l'après Deuxième Guerre mondiale, où tout s'est américanisé, uniformisé, quand tout le monde se retrouve équipé « [...] du semblable uniforme couleur cachou, matriculé, proclamant sur tous les dos en sueur de la terre l'inégalable supériorité d'une race de producteurs standardisés » ( $V$, 20).

Mélancolie d'un " temps enfin arrêté, figé, vaincu » $(V, 32)$, d'un " temps mort opaque, stagnant " $(V, 91)$ où certains personnages, les joueurs de cartes de l'Hôtel par exemple, sont comme des «momies»:

Et il me semblait les voir : à demi momifiés, avec ces mégots comme momifiés eux aussi [...] et les gestes avares aussi, raides, lents, les mains de momies ramassant les cartes, les déployant lentement en éventail devant les visages de momies, les yeux de momies [...]. ( $V$, 32)

D'autres, comme les cinq arabes de la p. 39, ne sont plus que les résidus d'une autre histoire, celle des guerres coloniales, et désormais carrément « hors du temps supprimés, sortis des lointaines et ombreuses avenues de l'Histoire ».

\footnotetext{
${ }^{24}$ Walter Benjamin, op. cit., p. 191.

${ }^{25}$ Ibid., p. 151.
} 
Ou bien l'Histoire est arrêté, «[...] semblable à une sorte d'épais magma où l'instant serait comme le coup de bêche dans la sombre terre, mettant à nu l'indénombrable grouillement de vers " $(V, 162)$. Ou bien elle est dégradation :

[...] toute vie n'est, à partir des éphémères gloires de l'adolescence, qu'un acheminement de défaites en défaites jusqu'à la décrépitude finale, jusqu'au désastre final et définitif, ce pourquoi sans doute religions et philosophes s'efforcent de travestir la souffrance en bienfait et la pourriture finale en délivrance $[\ldots]$. $(V, 119-120)$

D'un côté comme de l'autre donc, L'Histoire, sans salut, n'offre plus au regard du spectateur que sa «facies hippocratica $[. .$.$] comme$ un paysage primitif pétrifié $»^{26}$.

Du temps ne reste que le " chuintement ", ce «bruit même du temps épuisé, harassé » $(V, 24)$ et l'Histoire n'est que désastre, mais un désastre sans apocalypse, sans rédemption ni relève messianique d'aucune sorte. D'où cette tendance, chez Claude Simon (tendance qui ne fera que s'amplifier dans les autres romans), à rabattre toute la narration sur un présent d'énonciation, plus exactement un « maintenant " parfaitement ambigu, sorte de passé rapporté au présent, un à présent qui n'est pas sans évoquer le « jetztzeit » benjaminien : "Maintenant, il (Montès) passait le plus clair de son temps [...]" (23), « Maintenant il y avait des lavandières agenouillées [...] (92), « Maintenant il pouvait les voir au grand jour [...] » (93), «Et maintenant, assis à côté de Montès [...] " (188). La " fin » du roman est très claire sur ce point, qui souligne l'absence de fin, de terme, d'accomplissement, tout étant condamné « à s'épuiser sans fin, sans espoir de fin » $(V, 191)$.

Une fois expulsée toute conception du temps et de l'Histoire protensive, orientée, signifiante, nous voilà ramenés au pur battement vie/mort du présent qui ne s'aborde peut-être que dans cette rhétorique de l'infigurable, qui semble le chiffre ultime de la mélancolie. 


\section{La rhétorique de l'infigurable mélancolie}

\section{La loi de la syncope et de l'oxymoron}

L'oxymoron est baroque. Dans sa dissociation condensée, dans son assimilation condensée des contraires, il est la figure privilégiée de la Contre-Réforme qui associe et rend non contradictoires «la présence réelle du Christ en l'hostie » et "l'évidente absence physique de la personne $»^{27}$.

Par ailleurs, la scansion vie/mort, dans sa pureté oxymorique proche de la syncope, débouche sur la mélancolie de l'irreprésentable. La mort de Rose pour Montès est donc tragique mais d'un tragique sans dramaturgie, sans rituel ni cérémonial, sans acheminement, dénouement, fin, mais simplement « interruption » $(V, 142)$.

De là encore ces fréquents télescopages qui sont plus que de simples oppositions. Télescopages d'ordre et de désordre annoncés dés l'épigraphe du roman ${ }^{28}$ : Montés, dans le désordre de sa vie, va " à contre-courant » $(V, 83)$ de l'ordre mortifère de la société momifiée dans laquelle il a fait intrusion. Aussi étroitement associées qu'incompatibles, la terre et la guerre, l'homme et la femme : la terre, dans son "ingestion du guerrier» (79), le couple, dans leur " impossible et contradictoire tentative de se nier et de se survivre " (75).

Montés lui-même, véritable oxymoron incarné, en proie à $l^{\prime} \ll$ obsession $[\ldots]$ de son contraire, comme les voleurs ont l'obsession de l'honnêteté, les putains de la respectabilité » $(V, 116)$, est le fruit d'« un homme et [d']une femme qui n'étaient pas faits pour se comprendre, s'étaient néanmoins suffisamment entendus pour, en une seule nuit d'accouplement, l'engendrer, après quoi chacun était reparti de son côté » (56-57).

Vie/Mort, ces deux pôles de la vie indissociablement liés, sont rejoués dans la simultanéité et sur le mode très complexe (œdipienfaulknérien-beckettien) lors de la mort de Rose, vécue par Montés comme un viol incestueux et, toute temporalité abolie, comme une deuxième naissance, autrement dit une deuxième expulsion dans

\footnotetext{
${ }^{27}$ Christophe Deshoulières, "Narcisse et le Saint Esprit », in Résurgences baroques, La Lettre volée, «Essais », 2001, p. 202.

${ }^{28}$ " Deux dangers ne cessent de menacer le monde : l'ordre et le désordre. P. Valéry ».
} 
le vide du monde. Toute la page 146 serait à relire dans le détail pour voir à quel point est violent ce télescopage Vie/Mort. Télescopage des temps (la jeune femme devient son aînée), télescopage des personnes (la mère/l'amante), télescopage des sentiments (à l'horreur d'une expulsion, " hurlant et terrifié », dans le monde, le bonheur aussitôt avorté d'une venue au monde qui n'est que venue au « vide »):

[...] il se trouvait ramené à un état en quelque sorte fotal, lové dans la douloureuse et torturante (dit-on) quiétude d'une vie intrautérine dont il allait être - pour la seconde fois, et pour la seconde fois d'entre les cuisses d'une femme, bien que celle-ci fût de cinq ans plus jeune que lui - expulsé, projeté, hurlant et terrifié, dans le vide. $(V, 146)$

C'est bien le deuil originaire, mélancolie première, ontologique, qui se rejoue ici, dans cette rageuse et superbe page, où le narrateur pourrait déjà se dire, usant d'un oxymoron à venir, "bâillonné et hurlant $»$.

Dans cette contradiction d'une parole à la fois impuissante à s'exprimer et hyperboliquement proférée, crucifiée entre bavardage et mutisme, réside l'aporie mélancolique et sa réponse profuse, dans la multiplicité de ses replis baroques.

\section{Lepli baroque de l'écriture et de la voix mélancoliques}

Pour Deleuze, dans la pensée baroque, l'objet n'existe pas en tant que tel et n'est que « la déclinaison de ses profils». Le Baroque ne renvoie pas à une essence, mais plutôt à une fonction opératoire, à un trait. Il ne cesse de faire des plis : « Le trait du baroque, c'est le pli qui va à l'infini. ${ }^{29}$. De même, tout se replie dans le roman de Claude Simon.

Le titre déjà, Le Vent, qui se replie sur son sous-titre : Tentative de restitution d'un retable baroque. L'idée d'articulation et de pliure qui est le propre du " retable », on la retrouvera jusque dans l'évocation de la sexualité, exprimée en termes d'« ajustage » et de

\footnotetext{
${ }^{29}$ Gilles Deleuze, Le Pli, Éditions de Minuit, «Critique », p. 5. Relevons par ailleurs, dans cet ouvrage, cette phrase qui fait si bien écho au thème, au propos et au titre du roman de Claude Simon : «Le pli est inséparable du vent » (p. 43).
} 
" travail de serrurerie " !... $(V, 90)$. Autrement dit, la "modulation » a remplacé le "moule » et s'est substituée à la dualité forme-matière, sujet-objet, d'emblée réfutée par Claude Simon au motif que la réalité n'est pas « douée d'une vie propre, superbe, indépendante de nos perceptions " (4). La modulation du pli est aussi la figure privilégiée de la mélancolie : tronc plié d'aulne verlainien, pli de la réflexion, du spéculaire mélancolique ${ }^{30}$, pli du dédoublement narcissique et identificatoire du couple Montès/narrateur dont on trouve tant d'exemples dans la peinture baroque (pensons simplement au Narcisse de Caravage, au «Iste ego sum » de celui de Poussin).

Montès, personnage principal, n'est rien d'autre finalement que le produit d'une imagination (celle du narrateur) qui se replie sur des paroles (celles du notaire) : «Et je cherchais à l'imaginer, comme le décrivait le notaire $[\ldots] »(V, 8)$. Il n'est que d'être pris dans les plis de paroles qui se déplient en ondes concentriques et le constituent pur objet de chronique et de rumeurs. Une phrase déplie exactement un tel repli identificatoire : « [...] tandis que le notaire parlait, donc, je ne pouvais m'empêcher d'imaginer l'autre, celui qui avait ainsi défrayé la chronique de la ville et dont les gens comme le notaire n'avaient probablement pas encore fini de parler $[\ldots] »(4)^{31}$. D'où cette narration cahotante, tâtonnante, ramifiée, sinueuse, d'une histoire à la fois parfaitement accomplie et impossible à rétablir, à remettre à son hypothétique place première, brefà restituer, encore davantage produite que reproduite par les diverses voix qui la récitent ou peut-être l'imaginent, ce qui donne au roman ce ton particulier de récitatifmélancolique ou de " choeur antique », celui de la voix « medium » du notaire par exemple (83).

Le temps lui-même n'est plus seulement ou suspendu ou dégradé, il est encore, au rythme des saisons, replié, spiralé, "se télescopant, s'immobilisant ou se dilatant tour à tour " $(V, 114)$, et pour finir, là encore hors temps, comme dans ces vers de T. S. Eliot \{Four Quartets) que Claude Simon mettra en épigraphe à son roman L'Acacia :

${ }^{30}$ Cf. Jean Starobinski, La Mélancolie au miroir, « Trois lectures de Baudelaire », Julliard, 1989.

31 Souligné par nous. 
Time present and time past

Are both perhaps present in time future, And time future contained in time past.

Mélancolie héraclitéenne d'une temporalité circulaire et arrêtée, dépliée, repliée sur le présent, sinon la présence dont Montès semble destiné à ne connaître que son envers, dans ses absences contemplatives et douloureuses après la mort de Rose. Il s'abîme dans un rais de soleil sur le mur pour sentir, non pas l'éternité bien sûr, mais le " temps » lui-même, "l'éternel recommencement, l'éternel cheminement de la matière inerte, insensible, tournant dans l'infini $[. .$.$] »$ $(V, 156)$.

Le Baroque, pour Bonnefoy ${ }^{32}$, à l'image du Baldaquin du Bernin à St Pierre de Rome, est ce qui déploie la durée humaine «mais en même temps la recourbe, comme en spirale, dans l'unité du divin ", il est donc bien le pli, mais recourbé sur l'Un. Ainsi le corps convulsionné de la Sainte Thérèse du Bernin sur le point de se replier sur la flèche d'or dont l'ange s'apprête à lui transpercer le cœur. Le pli baroque de la narration simonienne, lui, ne se replie plus que sur l'absence. Elle est faite d'emboîtements et d'accumulations, de digressions et de commentaires auctoriaux mêlés d'approximations, profits, jaillissant en jets d'eau baroques, à l'image, peut-être, de ce que Marc Fumaroli appelle "poétique ignatienne ", à l'image, plus sûrement, de la profusion baroque d'un cadre qui n'encadre plus rien, tel ce cadre extravagant du Palazzo Barberini autour d'une absence de peinture ${ }^{33}$. Très exactement à l'image de «ce minimum de formes extérieures faute de quoi, probablement, comme une vieille baraque pourrie et privée de ses étais, notre monde s'effondrerait, basculerait en quelques instants dans le vide et le néant » $(V, 13)$.

Tant de replis vers le cadre de la narration, tant d'insistance à la référer aux sources diverses de son émission et surtout pas à quelque " référent " de la réalité, ne font que confirmer la signature baroque de l'œuvre (ici, le roman), qui ne devient, selon l'expression de Severo Sarduy, qu'une "mise en scène de sa machinerie ", la " somme des citations et les émissions multiples de voix » réfutant « l'existence

\footnotetext{
${ }^{32}$ Yves Bonnefoy, Rome 1630, Flammarion, "Champs », 2001, p. 34.

${ }^{33}$ Cf. Christophe Deshoulières, op. cit., p. 205.
} 
d'un centre émetteur un et naturel $»^{34}$. Autrement dit, prenant acte de l'infigurable.

\section{La mélancolie de l'infigurable}

Le centre n'est plus chez Claude Simon que ce résidu d'un ancien souffle divin, désormais matérialisé en vent "sauvage et froid" $(V, 8)$, abrutissant (19) ; il n'est, avec la lumière, qu'une « absence de quelque chose " (57) qui rend Montès mieux encore que "décharné », " désincarné » (58). Désincarné: voilà qui rappelle qu'inversement le Baroque est le règne de l'incarnation dans l'image, dans le "devenir image de l'infigurable ${ }^{35}$. D'où ces références, sans doute parodiques chez Simon, à une esthétique baroque mais totalement désacralisée, désincarnée et du coup assez grotesque. Ainsi de ces

[...] deux saintes tordant leurs mains parmi les tourbillons pétrifiés de leurs voiles sous l'impalpable et poussiéreux linceul, leurs visages voluptueux et peints ruisselants de larmes peintes, insensibles, défaillantes, pâmées dans le feu d'une éternelle souffrance, d'une éternelle extase. $(V, 154)$

Quant au théâtre du monde, il n'a plus rien à voir avec le « theatrum mundi » d'un «Lope de Vega » ou d'un " Calderon ». Il est réduit à « quelque chose de muet », la scène « seulement occupée par le vent " $(V, 170)$. On voit donc l'infigurable ou bien défiguré, réduit à sa caricature, à sa grimace, ou bien réduit au silence. Les églises de la ville flottent « la quille en l'air », emportant

[...] leurs vierges poignardées, debout dans leurs somptueuses robes de douleur, tordant leurs doigts chargés de diamants, levant leurs yeux aux pleurs de diamants vers leur fils supplicié, aux pieds polis par les lèvres des amoureuses et des enfants, les reins ceints de dentelle immaculée, nu, noir, et juif. $(V, 30)$

C'est tout le mystère de l'incarnation qui est lui-même réduit à cette dérisoire et emphatique théâtralisation baroque, réduit au geste de sa démonstration.

${ }^{34}$ Severo Sarduy, Barroco, Gallimard, «Folio essais », p.83.

${ }^{35}$ Marie-José Mondzain, L'image peut-elle tuer? Bayard, 2002, p. 32. 
L'infigurable donc, comme ce «barbouillage dépourvu de toute signification " $\{V, 139)$, dans " cette histoire de Balzac », Le Chefd'œuvre inconnu, auquel Claude Simon fait allusion par une périphrase. L'infigurable, voilà bien ce qui constitue le point aveugle mais essentiel du propos de Claude Simon. Sur fond de retrait de toute présence, le retrait de toute représentation y compris romanesque. "Mais n'allez pas vous figurer... - Je ne me figure rien. Du moins j'essaye de ne rien me figurer. " $(98)^{36}$ : ces phrases ambiguës résonnent étrangement alors, et d'autant plus étrangement que Montès vient de recevoir en pleine figure le violent coup de poing du boxeur !...

Retrait de toute présence. De fait, il y a beau temps que les dieux se sont retirés, et Montés qui si souvent dans le roman frôle la figure christique, qui finit " assis sur ce banc » $(V, 190)$, comme une sorte de Christ aux outrages, est un fils interdit de tout accès au Père, bien vite exclu du Patrimoine, à qui de surcroît la paternité, fûtelle par procuration (les filles de Rose, dans un orphelinat après la mort de leur mère) est interdite. S'il évoque bien la figure christique pourtant, c'est une figure christique vaine et incongrue, parfois grotesque voire clownesque, dans un monde définitivement sans transcendance. D'où cette peinture du Christ qu'il regarde dans le parloir de l'orphelinat : une scène totalement désacralisée, totalement vidée de mystère et de sens : «[...] quelque chose avec des femmes debout dans des voiles bleus, et des clous, et des gouttes de sang habilement peintes sur des pieds percés, et un ciel noir » $(V, 183)$.

Reste alors l'essentiel qui lui est exactement contigu : « [...] le jardin, la lumière poudroyante, le monde extérieur brûlant, embrasé. » $\{V, 183)$.

Reste « le vieux monde souillé sans cesse resurgissant à chaque aube dans son originelle virginité sous l'éclatante lumière, sans mystère, évident $"(V, 190)$.

Reste le monde de l'évidence et celui de l'exigence. Exigence morale dont Kant fait le propre de la mélancolie. D'où le souci de Montès : « Mais est-ce qu'il est impossible de faire un seul geste [...] sans que le mal... sans que tout se trouble, comme la vase d'une mare $[\ldots] »(V, 121-122)$. 
Pour finir, le narrateur lui demande s'il ne lui faudrait pas " rentrer dans un couvent" $(V, 186)$, pour être mis " hors d'état de nuire ", ne plus être un "vivant reproche" ? Autrement dit : ne plus toucher à rien?

\section{Conclusion : éluder les apories du tact}

Que reste-t-il, une fois enregistrée l'impossibilité de toute Figure et de tout figurable, si ce n'est le pli du repli de la trace et du vestige, si ce n'est le pli de l'écriture simonienne qui commence, à partir de ce roman, à se replier (ou à se déplier) sur la pure description de l'évidence, tenant compte patiemment, " mot à mot $\|^{37}$, du «branle perpétuel » montanien, de «l'humaine condition"?

Profonde est simplement la mélancolie de l'apparaître qui ne peut se faire, stylistiquement, que sur le mode biaisé du pli de la comparaison, "sous l'espèce d'un comme qui oblitère son comme tel $»^{38}$. Il n'est pas de mot propre, et le langage originellement métaphorique suscite transport, déplacement à l'infini. La mélancolie de l'écriture simonienne dans Le Vent, n'est que d'être hantée par le silence auquel elle n'échappe que pour y retourner et duquel elle est encore relancée vers la parole. Profusion baroque des plis de l'écriture, multiplication des perspectives sans espoir de perspective unique réalisant la présence. La mélancolie du fragment d'ailleurs, rappelons le, tient moins au fait qu'il serait ruine d'une unité première effondrée, que ruine d'une unité première toujours déjà effondrée, que ruine d'une impossible unité, qui n'a jamais commencé, qui ne finira jamais, tel ce

[...] mur gris sans commencement ni fin, avec ses vieilles affiches déchirées aux pans soulevés par le vent, leurs couleurs fanées, ou quelquefois encore vives, criardes, leurs couleurs délavées, leurs fragments de textes sans commencement ni fin non plus, sans suite, se juxtaposant, se contredisant [...]. $(V, 116)$

Bref, à l'image même de la mélancolique nuit de l'existence, de la parole qui tente vainement de la dire, de son texte et de son com-

\footnotetext{
${ }^{37}$ Cf. le titre de la conférence de Claude Simon, "La fiction mot à mot» (1972).

${ }^{38}$ Cf. Jacques Derrida, "Le retrait de la métaphore », in Psyché, Galilée, 2003.
} 
mentaire, condamnés eux aussi, comme ce vent d'automne qui gémit dans la nuit, à « [...] s'épuiser sans fin, sans espoir de fin»(191).

Resterait peut-être alors, en guise d'épigraphe finale à l'étude de " la mélancolie Le vent» que nous avons tenté de faire, ces quelques vers de T. S. Eliot :

Men and bits of paper, whirled by the cold wind That blows before and after time.

(« Hommes et bouts de papier tourbillonnant dans le vent froid Qui souffle avant et après le temps »)

(Quatre quatuors) 